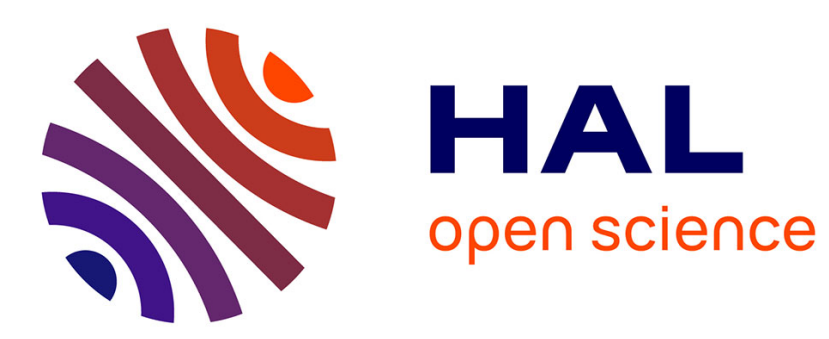

\title{
Electrovibration Signal Design
}

Zlatko Vidrih, Eric Vezzoli

\section{To cite this version:}

Zlatko Vidrih, Eric Vezzoli. Electrovibration Signal Design: A Simulative Approach. Haptics: Perception, Devices, Control, and Applications, Jul 2016, London, United Kingdom. hal-01341974

\section{HAL Id: hal-01341974 \\ https://hal.inria.fr/hal-01341974}

Submitted on 5 Jul 2016

HAL is a multi-disciplinary open access archive for the deposit and dissemination of scientific research documents, whether they are published or not. The documents may come from teaching and research institutions in France or abroad, or from public or private research centers.
L'archive ouverte pluridisciplinaire HAL, est destinée au dépôt et à la diffusion de documents scientifiques de niveau recherche, publiés ou non, émanant des établissements d'enseignement et de recherche français ou étrangers, des laboratoires publics ou privés. 


\title{
Electrovibration Signal Design A Simulative Approach
}

\author{
Zlatko Vidrih $^{1}$ and Eric Vezzoli ${ }^{2}$ \\ 1 Zienkiewicz Centre for Computational Engineering, College of Engineering, \\ Swansea University, Bay Campus, Fabian Way, Swansea SA1 8EN, United Kingdom \\ z.vidrih@swansea.ac.uk \\ 2 L2EP, University Lille1 F59650 Villeneuve dAscq, France \\ eric.vezzoli@ed.univ-lille1.fr
}

\begin{abstract}
Electrovibration technique can modify user's perception of a surface through the modulation of the sliding friction accordingly to the voltage applied. This paper is introducing a novel approach to virtual haptic rendering in electrovibration based haptic displays in order to provide realistic feeling of a simulated surface, where the required voltage signal is obtained using a simplified equation. The approach was validated by the use of a finite element computational framework able to simulate tactile scenarios on real and virtual surfaces. A database of precompiled tactile scenarios was generated to predict outputs for custom parametric surfaces through a conditional average estimator method. In addition, an experimental database obtained by active exploration of different surfaces, is utilised for texture rendering. A web application, comprising the algorithms described in the paper, has also been developed, and is freely available to use at http://www.haptictexture.com.
\end{abstract}

Keywords: electrovibration · haptic rendering - finite element model . finger pad · virtual prototyping · haptic display · friction

\section{Introduction}

Since the beginning of the surface haptic era efforts have been made to develop an effective method to stimulate the finger pad. A lot of attention has been given to hardware development and optimisation in order to show the potential advantages the electrostatic attraction technology could bring in the humanmachine interaction [1]. However, only little effort was pursued on the design of the realistic surface haptic stimuli.

The most common techniques for friction modulation in haptic displays are electrovibration $[2,3]$, which is the topic of the paper, and ultrasonic vibrations [4]. In order to generate the high fidelity stimuli, two important features of tactile recognition should be recalled: Robles-De-La-Torre and Hayward [5] demonstrated that, for shape recognition, the direction of the applied force on the fingertip is more important, than the actual geometry, and later Wiertlewski 
and Hayward [6] observed, that the spatial spectrum of the skin displacements can encode tactual texture. A stimulus design aiming to mimic the real interaction with everyday objects should therefore take advantages of both features to provide a more natural interaction with surface haptic devices.

The aim of current research is to develop a freely available computational environment for tactile signal design for haptic displays to simulate real surfaces. A virtual haptic rendering algorithm is introduced, where friction profiles are used as an input for control of electrovibration displays. These profiles are obtained from measured friction data or FE simulations for different reference standards (RS), i.e. reference surfaces. Since FE analyses can take several minutes to several hours to complete, or even several days in case of rigorous $3 \mathrm{D}$ analysis, they can not be used in real-time haptic rendering. Therefore, a non-parametric approach is suggested here to perform a real-time electrovibration stimuli design. This approach is based on the conditional average estimator (CAE) method, which is a kind of probabilistic artificial neural network with a multi-dimensional non-parametric regression [7]. The method enables relatively simple empirical modelling of different physical phenomena, provided that the sufficient experimental data is available.

The paper is organized as follows, in Sect. 2 texture synthesis algorithms used in the study are introduced, and in Sect. 3 their application is demonstrated on simple example. In Sect. 4 the developed on-line tool, based on the described algorithms, is presented. The paper concludes with the discussion of the results and options for future work.

\section{Texture Synthesis Algorithms}

A short review of related work is firstly presented (Sect. 2.1), then the principles of electrovibration modelling are recalled (Sect. 2.2), and friction-applied voltage relation is introduced (Sect. 2.3). Later, the algorithm applied to an experimental friction database or to friction data provided by the computational framework is given (Sect. 2.4), with a description of the CAE method, as applied in current study.

\section{$2.1 \quad$ Related work}

Kim et al. [8] have observed, that despite the great user interaction opportunities haptic displays can provide, there has been little work done on design and evaluation of algorithms for generating rich tactile sensations. Therefore they have proposed an algorithm for rendering of 3D bumps based on the gradient of the simulated surfaces and a friction model based on psychophysical experiments.

A static method based on grey-scale levels of the displayed image, i.e. applied voltage (friction) at a location on a screen is proportional to the grey-scale value of pixels, is also widely used, as it is intuitive and relatively easy to implement. Radivojevic et al. [1] proposed an approach using the image segmentation method, where the photos, taken by the user, are automatically broken into a 
set of areas defined by the visual content (faces, hair, clothes, sky, land or water) and different textures are automatically assigned to different regions of the image.

Other option for texture rendering consist of record-and-re-play technique, in which a recorded values of quantities (forces, displacements, accelerations, etc.) while exploring a real surface, are re-played when exploring a virtual surfaces. A repository of 100 haptic texture models for use by the research community Haptic Texture Toolkit (HaTT) is available at the Penn University web page [9].

A similar approach is used here, albeit for the finger-screen interaction, where friction profiles are either recorded experimentally or obtained using the finite element analysis of tactile scenarios (Sect. 2.4). An extensive database of friction profiles for simple RSs has been established and implemented in an probabilistic artificial neural network in an online tool HapTex [10].

\section{$2.2 \quad$ Electrovibration modelling}

Electrovibration tactile displays exploit the Coulomb electrostatic force between the finger pad and an insulated high voltage supplied conductive plate on the screen to modulate friction. When the electric current is off, the percieved friction $F_{\mathrm{t}}$ while sliding the finger pad on a screen is proportional to the normal load $F_{\mathrm{n}}$, i.e.

$$
F_{\mathrm{t}}=\mu F_{\mathrm{n}}
$$

if a Coulomb friction law, with a constant friction coefficient $\mu$, is assumed. If voltage is applied (AC) the perceived friction is increased due to the increase of normal force by the electrostatic force $F_{\mathrm{e}}$, with friction coefficient $\mu$ remaining unchanged:

$$
F_{\mathrm{t}}=\mu\left(F_{\mathrm{n}}+F_{\mathrm{e}}\right)
$$

By assigning a different friction value to locations $\mathbf{r}=(x, y)$ of the screen, i.e. $F_{\mathrm{t}}=f(\mathbf{r})$, different RS, i.e. reference textures, can be represented ${ }^{3}$. Here we utilize the expression for the electrostatic force between the finger pad and the tactile display as proposed by Kaczmarek et al. [11], i.e.

$$
F_{\mathrm{e}}(\mathbf{r})=\frac{\epsilon_{0} A U(\mathbf{r})^{2}}{2\left(\frac{d_{\mathrm{sc}}}{\epsilon_{\mathrm{sc}}}+\frac{d_{\mathrm{i}}}{\epsilon_{\mathrm{i}}}\right)\left(d_{\mathrm{sc}}+d_{\mathrm{i}}\right)},
$$

where $A$ is contact surface area, $U(\mathbf{r})$ the voltage applied at location $\mathbf{r}, \epsilon_{0}$ permittivity of vacuum, and $\epsilon$ and $d$ relative permittivity and thickness of the assumed dielectrics (sc - stratum corneum, i.e. the outermost layer of the skin, and i the screen insulator), respectively. Equation (3) gives better approximation of experimentally measured electrostatic forces [12], and is more widely used [13, 1], than the expression proposed by Strong and Troxel [3].

\footnotetext{
${ }^{3}$ Actual friction values at location $\mathbf{r}$ are time, pressure and velocity dependant, but
} this is neglected in notation here. 


\subsection{Friction-Voltage Relation}

In order to mimic the friction force profile of the RS, similar friction force cues have to be applied on the surface of a haptic display (HD), i.e. $F_{\mathrm{t}}^{\mathrm{RS}}(\mathbf{r}) \simeq F_{\mathrm{t}}^{\mathrm{HD}}(\mathbf{r})$, where

$$
F_{\mathrm{t}}^{\mathrm{RS}}(\mathbf{r})=\mu^{\mathrm{RS}}(\mathbf{r}) F_{\mathrm{n}}^{\mathrm{RS}}
$$

and

$$
F_{\mathrm{t}}^{\mathrm{HD}}(\mathbf{r})=\mu^{\mathrm{HD}}\left(F_{\mathrm{n}}^{\mathrm{HD}}+F_{\mathrm{e}}(\mathbf{r})\right) .
$$

Postulating $F_{\mathrm{n}}^{\mathrm{HD}}=F_{\mathrm{n}}^{\mathrm{RS}}=F_{\mathrm{n}}$, i.e. the normal load is approximately constant during the exploration ${ }^{4}$, and equating (4) and (5)

$$
F_{\mathrm{e}}(\mathbf{r})=\left[\frac{\mu^{\mathrm{RS}}(\mathbf{r})}{\mu^{\mathrm{HD}}}-1\right] F_{\mathrm{n}}
$$

is obtained. Then the required voltage $U(\mathbf{r})$ at position $\mathbf{r}$ can be expressed from (3) and (6) as

$$
U(\mathbf{r})=\sqrt{\frac{2\left(\frac{d^{\mathrm{sc}}}{\epsilon^{\mathrm{sc}}}+\frac{d^{\mathrm{i}}}{\epsilon^{\mathrm{i}}}\right)\left(d^{\mathrm{sc}}+d^{\mathrm{i}}\right)\left[\frac{\mu^{\mathrm{RS}}(\mathbf{r})}{\mu^{\mathrm{HD}}}-1\right] F_{\mathrm{n}}}{\epsilon_{0} A} .}
$$

Due to the relatively low force levels present in typical screen exploration $(<0.5 \mathrm{~N})$ a linear relation ${ }^{5}$ can be assumed between the finger pad contact area and the normal force, i.e. $A=k F_{\mathrm{n}}$, which leads to a simplified expression

$$
U(\mathbf{r})=\sqrt{\frac{2\left(\frac{d^{\mathrm{sc}}}{\epsilon^{\mathrm{sc}}}+\frac{d^{\mathrm{i}}}{\epsilon^{\mathrm{i}}}\right)\left(d^{\mathrm{sc}}+d^{\mathrm{i}}\right)\left[\frac{\mu^{\mathrm{RS}}(\mathbf{r})}{\mu^{\mathrm{HD}}}-1\right]}{\epsilon_{0} k}},
$$

which can be implemented in the haptic signal control, considering the device limitations. If only pattern reproduction is of interest, as suggested in [6], a simple algorithm exploiting the maximum device capacity can be employed:

$$
U(\mathbf{r})=U_{\max } \sqrt{\frac{\mu^{\mathrm{RS}}(\mathbf{r})-\min \left(\mu^{\mathrm{RS}}(\mathbf{r})\right)}{\max \left(\mu^{\mathrm{RS}}(\mathbf{r})\right)-\min \left(\mu^{\mathrm{RS}}(\mathbf{r})\right)}},
$$

i.e. minimum and maximum friction force are assigned with $0 \mathrm{~V}$ and the maximum feasible voltage $\left(U_{\max }\right)$, respectively.

\footnotetext{
${ }^{4}$ When sliding the finger pad over a RS, e.g. surface with a bump, the normal force also fluctuates, but this is captured here in the location dependent coefficient of friction.

${ }^{5}$ When higher normal force is anticipated, a power law, e.g. as used by Adams et al. [14], should be employed. Provided, that the haptic display is able to determine the contact area, approximation is unnecessary.
} 


\subsection{Input Friction Data}

The spatial friction profile $\mu^{\mathrm{RS}}(\mathbf{r})$ in (7), (8), or (9), can be obtained either by computer simulations, or by measurements of friction $F_{\mathrm{t}}^{\mathrm{RS}}(\mathbf{r})$ and normal force $F_{\mathrm{n}}^{\mathrm{RS}}(\mathbf{r})$, e.g. by tribological experiments. Following, three examples of input friction data preparation are reported.

Experimental database. The experimental database used in this work was obtained in active touch experiments reported by Klöcker et al. [15]. The database comprises twelve materials: sandpaper (P24), sponge, latex, wax, linen, wood, plastic, aluminium, tile, tights, velvet and paper $\left(160 \mathrm{~g} / \mathrm{m}^{2}\right)$. Each material was stroked by seven participants ten times, i.e. there are seventy measurements per material. For more information about the database and experimental procedures see [15].

The force profiles $\left(F_{\mathrm{n}}^{\mathrm{RS}}(t), F_{\mathrm{t}}^{\mathrm{RS}}(t)\right)$ from the experimental database were re-sampled from $20 \mathrm{kHz}$ to $400 \mathrm{~Hz}$ to match the finger pad position sampling. Only the sliding parts of the experiments were used, where the assumption about constant normal force postulated in Sect. 2.3 is valid. Finally the friction profiles were re-sampled to location domain $\left(F_{\mathrm{t}}^{\mathrm{RS}}(\mathbf{r})\right)$. For $F_{\mathrm{n}}^{\mathrm{RS}}$ a mean value of $F_{\mathrm{n}}^{\mathrm{RS}}(\mathbf{r})$ during sliding was used. The database was also implemented in the web tool presented in section 4 [10], where a random selection from the experimental set is performed for calculation of voltage profile $U(\mathbf{r})$ of a selected material.

FEM simulations of tactile scenarios Previous finite element studies of human touch mostly include two or three dimensional modelling of finger pad exploration of real surfaces on tribological $[16,17]$ as well as neuro-mechanical levels or vibratory stimulations [18-20]. Abdolvahab [21] simulated the "squeeze film" effect, by comparing sliding of a finger pad over a real edge and a surface with varying friction coefficient.

The multi-physics computational framework used in this work is capable of simulating friction modulation due to electrostatic attraction directly from applied voltage signal in the haptic display, without changing the friction properties of the surface. Current work employs a 2D version of previously developed framework validated with experimental data $[20,17]$ as it is very useful for parametric studies in parallel to the computationally intensive 3D model. The framework was used here mainly in order to generate friction profiles $\mu^{R S}(\mathbf{r})$ for CAE database for three simple RS, i.e. a bump, an edge, and a sinusoidal surface, which can all be defined using three geometric parameters as shown in Fig. 1a. For each set of parameter values the finger pad was pressed against the surface $(0.25 \mathrm{~mm})$ and then slid parallel to the surface $(10 \mathrm{~mm})$ using different velocities. The 2D parametric FE model of the finger pad comprises six most characteristic subdomains to assure sufficient anatomical accuracy (Fig. 1b). The material and geometric properties obtained from literature [19] were used. Due to the small deformation range in the finger pad a visco-elastic material model was adopted. 
Typical model consisted of 3027 nodes and 2877 four-noded plane strain quadrilateral elements based on the standard isoparametric approach. The contact between the skin and the plate was modelled using penalty method with coulombic friction, where the RS are assumed rigid in comparison to the finger. More details on FEM analysis can be found at [10].

Conditional average estimator (CAE) As stated before, FE simulation are computationally intensive and cannot be applied in real-time applications. The CAE method can provide a real-time solution for a prescribed subset of surfaces. The CAE method [7] used in the study is a non-parametric empirical approach for the estimation of an unknown quantity as a function of known input parameters, provided that an appropriate database is available. In this work, the method was applied for the prediction of the friction force profiles $\mu_{R S}(\mathbf{r})$ for different RSs. CAE enables us to estimate the value of a function

$$
\mathbf{C}=f(\mathbf{B}, \mathbf{X})
$$

where $\mathbf{X}=\left\{\mathbf{X}_{1}, \ldots, \mathbf{X}_{n}, \ldots, \mathbf{X}_{N}\right\}$ represents a mathematical description of the phenomenon with a model matrix, i.e. a database, consisting of the finite number $(N)$ of model vectors $\mathbf{X}_{n}$, each attributed to a single scenario in an experiment. Each model vector is composed of two truncated vectors, i.e. $\mathbf{X}_{n}=\left\{\mathbf{B}_{n}, \mathbf{C}_{n}\right\}$, where $\mathbf{B}_{n}$ and $\mathbf{C}_{n}$ represent the input and output parameters of the $n^{\text {th }}$ experiment, e.g. in tribological experiment on grated surface $\mathbf{B}_{n}$ could consist of

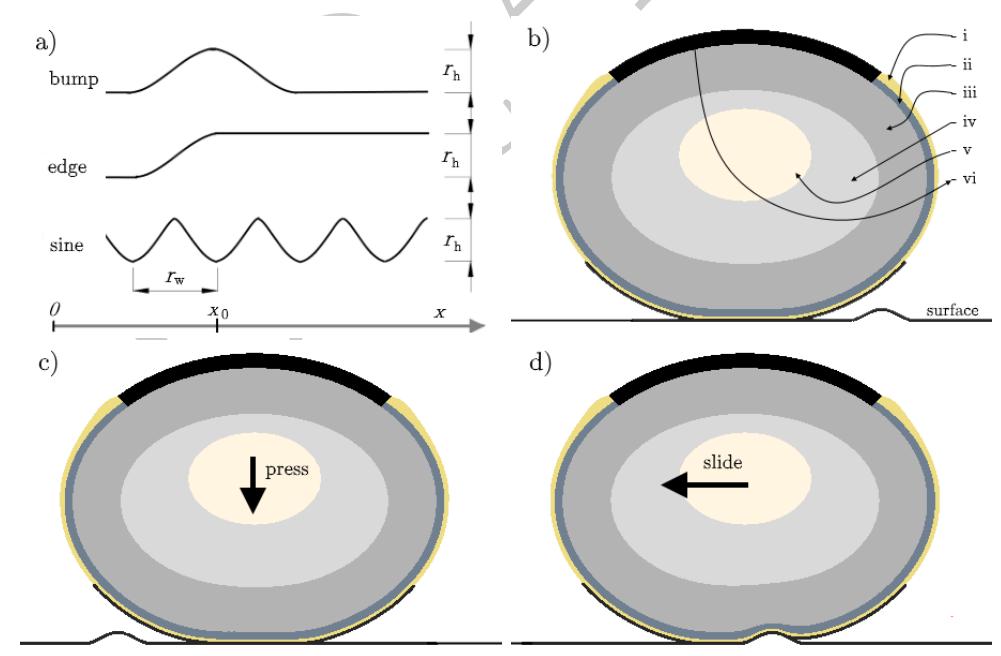

Fig. 1. a) Simulated surfaces: a symmetric bump, an edge, and a sinusoidal surface. Bump and edge are defined by the location of their maximum $\left(x_{0}\right)$, their width $\left(r_{\mathrm{w}}\right.$, and their height $\left(r_{\mathrm{h}}\right)$. Sinusoidal surface is defined by phase $\left(x_{0}\right)$, period $\left(r_{\mathrm{w}}\right)$, and amplitude $\left(r_{\mathrm{h}}\right)$.; b) FEM subdomains (i) stratum corneum, (ii) vital epidermis, (iii) dermis, (iv) hypodermis, (v) bone, and (vi) nail; c) deformed shape after pressing the finger towards the surface; d) deformed shape while sliding over the bump. 
normal force, velocity, and grating period, etc. and $\mathbf{C}_{n}$ could consist of measured quantities (observations), as maximum friction force, time and/or frequency response, etc. Scenario is described by a number of variables, which are treated as components of a model vector $X_{n}=\left\{b_{n 1}, \ldots, b_{n l}, \ldots, b_{n D}, \ldots, c_{n 1}, \ldots, c_{n k}, \ldots, c_{n M}\right\}$. Similarly vectors $\mathbf{B}$ and $\mathbf{C}$ represent the prediction vector $\mathbf{X}_{\mathrm{p}}=\{\mathbf{B}, \mathbf{C}\}$, i.e. the given truncated vector $\mathbf{B}$ and the unknown complementary vector $\mathbf{C}$. Our aim is therefore to estimate an unknown complementary vector $\mathbf{C}$ from a given truncated vector $\mathbf{B}$ and the model matrix $\mathbf{X}$, e.g. to predict the friction profile using (4), i.e. $\mu^{R S}(\mathbf{r})=F_{\mathrm{t}}^{R S}(\mathbf{r}) / F_{\mathrm{n}}^{R S}$, given the surface parameters, e.g. location of a bump, $x_{0}$, and its geometry, $b$ and $h$ (Fig. 1a).

The optimal estimator for the given problem can be expressed using the conditional probability density function:

$$
c_{k}=\sum_{n=1}^{N} \frac{a_{n} c_{n k}}{\sum_{n=1}^{N} a_{i}} .
$$

Here, an approach, used by Perus et al. [7], is utilised, i.e. a "non-constant width" Gaussian density function, centred at each model vector, to get its influence on the prediction vector, is chosen as a weight function:

$$
a_{n}=\frac{1}{(2 \pi)^{D / 2} \prod_{l=1}^{D} w_{n l}} \exp \left[-\sum_{l=1}^{D} \frac{b_{l}-b_{n l}^{2}}{2 w_{n l}^{2}}\right] .
$$

Variable $c_{k}$ is an estimate of the $k^{\text {th }}$ output variable, $c_{n k}$ is the same output variable corresponding to the $n^{\text {th }}$ model vector in the database, $N$ is the number of model vectors in the database, $b_{n l}$ is the $l^{\text {th }}$ input variable of the $n^{\text {th }}$ model vector in the database (e.g. $b_{n 1}, b_{n 2}, b_{n 3}, \ldots, b_{n L}$ ), and $b_{l}$ is the $l^{\text {th }}$ input variable corresponding to the prediction vector. The $D$ is the number of input variables, which defines the dimension of the sample space. Different values of $w_{n l}$ correspond to $l^{\text {th }}$ input variable for each model vector $\left(\mathbf{X}_{n}\right)$ from the normalized database [0 1]. See Perus et al. [7] for more details.

\section{Numerical examples}

Here, the generation of the database using FEM is presented for a single tactile scenario on example RS (Sect. 3.1). Same example is then used for evaluation of the implemented CAE method (Sect. 3.2)

\subsection{Finite element analysis}

A number of analyses was performed on all three demonstration RSs with varying geometric parameters, i.e. $r_{\mathrm{w}}$ was varied from 0.5 to $2.5 \mathrm{~mm}$ with a $0.25 \mathrm{~mm}$ step, and $r_{\mathrm{h}}$ was varied from 0.1 to $0.5 \mathrm{~mm}$ with a $0.1 \mathrm{~mm}$ step at constant sliding velocity. Location of a bump $x_{0}$ can be considered later by moving the location of the stimulus. Typical (normalized) output in terms of $\mu^{\mathrm{RS}}(\mathbf{r})$ is presented in Fig. 2a. For more information about model parameters used in the analysis see FAQ section of the web tool [10]. Fig.2b depicts the required voltage at location $x$ obtained using (8) and (9). 

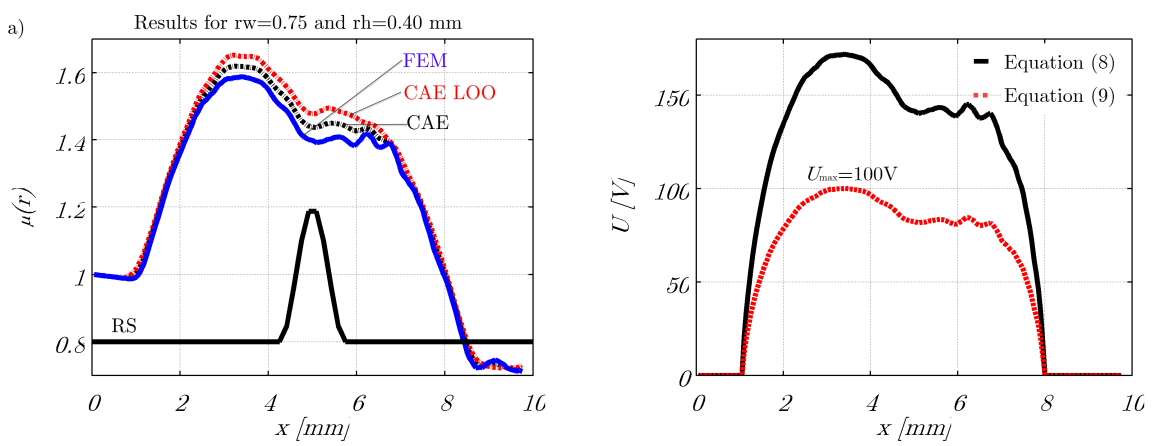

Fig. 2. A comparison of friction profiles obtained while sliding the finger pad over a bump using FEM, CAE with leave one out and CAE with full database (a), and the voltage profile $U(\mathbf{r})$ obtained using $(8)$, with $\epsilon^{\mathrm{sc}}=1650, \epsilon^{\mathrm{i}}=10, t^{\mathrm{sc}}=0.2 \mathrm{~mm}, t^{\mathrm{i}}=0.001$ $\mathrm{mm}$, and $k=200$, and using (9) with $U_{\max }=100 \mathrm{~V}\left(\mu^{\mathrm{HD}}=1\right)$

\subsection{CAE approximation}

After the generation of the database in the previous step - a "leave one out" (LOO) analysis was performed to asses the prediction quality and to optimise the weight parameters. The CAE output for input parameters present/absent in the database is depicted in Fig.2a, and compared to the actual FE results. Since the database is artificially generated and equally distributed, a relatively uniform smoothing $w$ is required, i.e $w_{\min }=w_{\max } \approx 0.13$ was determined using Matlab fminsearch function ${ }^{6}$. Now we can predict the friction profiles for similar surfaces, based on the precomputed results.

\section{Online Tool}

All previously described algorithms were implemented in a web application built using AJAX, i.e. asynchronous JavaScript, php, and XML, environment. The web tool provides the graphical user interface [10], which can be used across wide range of platforms, e.g. PC or mobile. Users can calculate haptic rendering of generic 2D surfaces based on templates, simulated in advance, or use their own friction profiles. The experimental database described in Sect. 2.4 [15] is also integrated. An example screenshot for bump simulation is presented in Fig. 3 .

\section{Discussion and Conclusion}

This paper introduce the haptic rendering algorithm based on FEM simulations, experimental data, and type of artificial neural network for generation of signals for RS not yet available in the database, but with similar properties as the known ones. Simple example RS using only three parameters are used for demonstration, although more complex surfaces can be implemented in the existing algorithms.

\footnotetext{
${ }^{6}$ Note that extreme extrapolation of results may lead to erroneous values.
} 


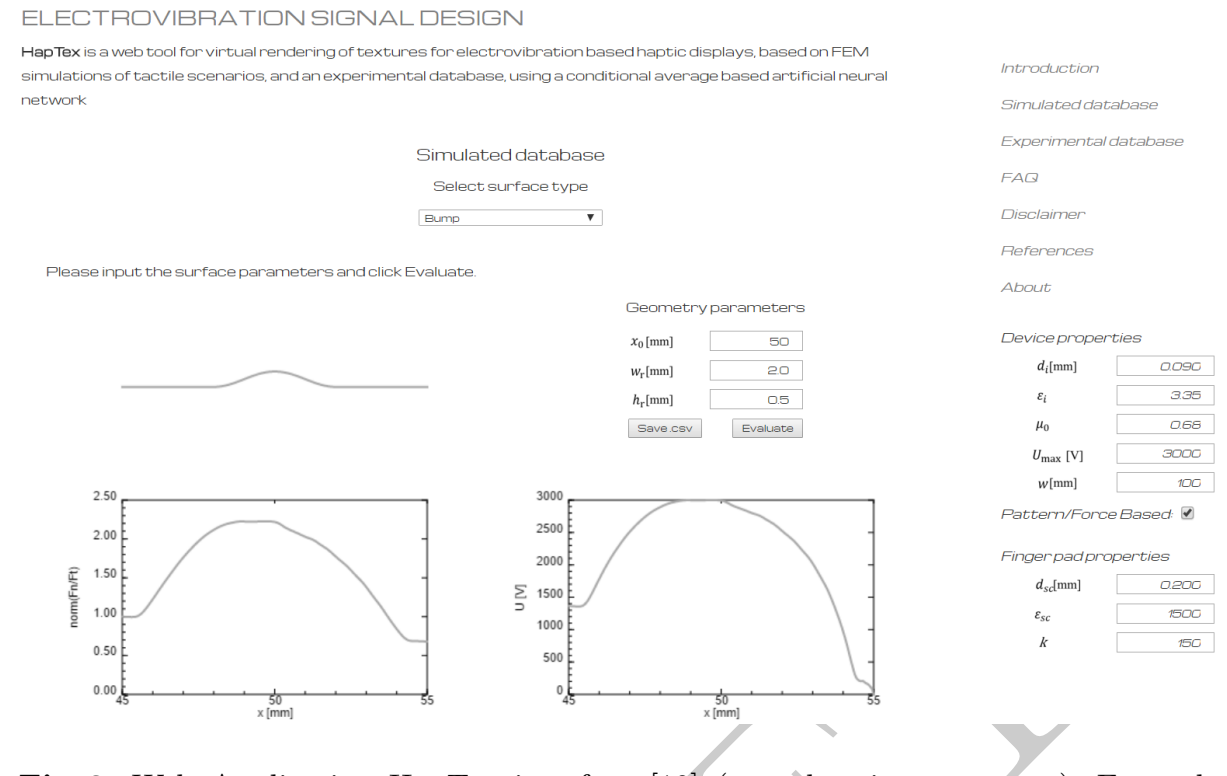

Fig. 3. Web Application HapTex interface [10] (www.haptictexture.com). Example bump simulation.

For demonstration purposes the method has been implemented in a freely accessible web tool, where users can try generating different voltage signals. Using the CAE methods the computational times shorten to milliseconds which makes the method feasible for real time haptic rendering. The accuracy of the predictions strongly depends on the database, however the database will be developed in time by adding virtual and real textures. In the future development the goal is to provide a downloadable library of textures for haptic surfaces, and although the present study deals only with electrovibration, the methods can be easily applied to ultrasonic and tactile displays combining both methods.

Acknowledgments. This work was supported by the European Union under the FP7 program FP7-PEOPLE-317100 Prototouch. We would like to express our gratitude to Dr. Anne Klöcker from the Institute of Neuroscience at Université catholique de Louvain, for providing us with the experimental database [15].

\section{References}

1. Radivojevic, Z., Beecher, P., Bower, C., Cotton, D., Haque, S., Andrew, P., Henson, B., Wall, S.A., Howard, I.S., Ingram, J.N., Wolpert, D.M., Salo, A.O., Xue, T.: 31.1: Invited paper: Programmable electrostatic surface for tactile perceptions. SID Symposium Digest of Technical Papers 43(1) (2012) 407-410

2. Mallinckrodt, E., Hughes, A.L., Sleator, W.: Perception by the skin of electrically induced vibrations. Science 118(3062) (1953) 277-278

3. Strong, R.M., Troxel, D.: An electrotactile display. Man-Machine Systems, IEEE Transactions on 11(1) (1970) 72-79 
4. Sednaoui, T., Vezzoli, E., Dzidek, B., Lemaire-Semail, B., Chappaz, C., Adams, M.: Experimental evaluation of friction reduction in ultrasonic devices. In: World Haptics Conference (WHC), 2015 IEEE. (2015) 37-42

5. Robles-De-La-Torre, G., Hayward, V.: Force can overcome object geometry in the perception of shape through active touch. Nature 412(6845) (2001) 445-448

6. Wiertlewski, M., Hayward, V.: Mechanical behavior of the fingertip in the range of frequencies and displacements relevant to touch. Journal of Biomechanics 45(11) (2012) 1869-1874

7. Perus, I., Poljansek, K., Fajfar, P.: Flexural deformation capacity of rectangular rc columns determined by the cae method. Earthquake Engng. Struct. Dyn. 35(35) (2006) 1453-1470

8. Kim, S.C., Israr, A., Poupyrev, I.: Tactile rendering of 3D features on touch surfaces. In: Proceedings of the 26th Annual ACM Symposium on User Interface Software and Technology. (2013) 531-538

9. Culbertson, H., Lopez Delgado, J., Kuchenbecker, K.: One hundred data-driven haptic texture models and open-source methods for rendering on 3d objects. In: Haptics Symposium (HAPTICS), 2014 IEEE. (Feb 2014) 319-325

10. : Haptic texture database. http://www.haptictexture.com Accessed: 2016-01-26.

11. Kaczmarek, K., Nammi, K., Agarwal, A., Tyler, M., Haase, S., Beebe, D.: Polarity effect in electrovibration for tactile display. Biomedical Engineering, IEEE Transactions on 53(10) (2006) 2047-2054

12. Vezzoli, E., Amberg, M., Giraud, F., Lemaire-Semail, B.: Electrovibration modeling analysis. In Auvray, M., Duriez, C., eds.: Haptics: Neuroscience, Devices, Modeling, and Applications. Volume 8619 of Lecture Notes in Computer Science. Springer Berlin Heidelberg (2014) 369-376

13. Giraud, F., Amberg, M., Lemaire-Semail, B.: Mêrging two tactile stimulation principles: electrovibration and squeeze film effect. In: World Haptics Conference (WHC), 2013. (2013) 199-203

14. Adams, M.J., Johnson, S.A., Lefèvre, P., Lóvesque, V., Hayward, V., Andró, T., Thonnard, J.L.: Finger pad friction and its role in grip and touch. Journal of The Royal Society Interface 10(80) (2012)

15. Klöcker, A., Wiertlewski, M., Théate, V., Hayward, V., Thonnard, J.L.: Physical factors influencing pleasant touch during tactile exploration. PLoS ONE 8(11) (11 2013) e 79085

16. Dandekar, K., Raju, B.I., Srinivasan, M.A.: 3-D finite-element models of human and monkey fingertips to investigate the mechanics of tactile sense. Journal of Biomechanical Engineering 125 (2003) 682-691

17. Vodlak, T., Vidrih, Z., Fetih, D., Peric, D., Rodic, T.: Development of a finite element model of a finger pad for biomechanics of human tactile sensations. In: Engineering in Medicine and Biology Society (EMBC), 2015 37th Annual International Conference of the IEEE. (2015) 909-912

18. Gerling, G.J.: SA-I mechanoreceptor position in fingertip skin may impact sensitivity to edge stimuli. Applied Bionics and Biomechanics 7(1) (2010) 19-29

19. Lesniak, D.R., Gerling, G.J.: Predicting SA-I mechanoreceptor spike times with a skin-neuron model. Mathematical Biosciences 220(1) (2009) 15-23

20. Vodlak, T., Vidrih, Z., Pirih, P., Skorjanc, A., Presern, J., Rodic, T.: Functional microanatomical model of Meissner corpuscle. In Auvray, M., Duriez, C., eds.: Haptics: Neuroscience, Devices, Modeling, and Applications. Volume 8619 of Lecture Notes in Computer Science. Springer Berlin Heidelberg (2014) 377-384

21. Abdolvahab, M.: Rendering edge enhancement tactile phenomenon by friction variation in dynamic touch. Journal of Biomechanics 44(1) (2011) 92-96 